\title{
Error Probability of DPSK Signals With Intrachannel Four-Wave Mixing in Highly Dispersive Transmission Systems
}

\author{
Keang-Po Ho, Senior Member, IEEE
}

\begin{abstract}
A semianalytical method evaluates the error probability of differential phase-shift keying signals with intrachannel four-wave mixing in a highly dispersive fiber link with strong pulse overlap. Depending on initial pulsewidth, the mean nonlinear phase shift of the system can be from 1 to 2 rad for signal-to-noise ratio penalty less than $1 \mathrm{~dB}$. An approximated empirical formula, valid for penalty less than $2 \mathrm{~dB}$, uses the variance of the differential phase of the ghost pulses to estimate the penalty.
\end{abstract}

Index Terms-Differential phase-shift keying (DPSK), fiber nonlinearities, intrachannel four-wave mixing (IFWM).

\section{INTRODUCTION}

$\mathbf{T}$ HE DIFFERENTIAL phase-shift keying (DPSK) signal has been widely studied recently for long-haul lightwave transmission systems [1]-[4]. In additional to the 3-dB receiver sensitivity improvement to on-off keying (OOK), the high-speed DPSK signal also has larger tolerance to fiber nonlinearities than OOK signal [5]. Most DPSK experiments use return-to-zero (RZ) short pulse and launch a constant-intensity pulse train with phase modulated to each RZ pulse.

For 40-Gb/s signal in dispersive fiber, each RZ pulse broadens very fast by chromatic dispersion and overlaps with each other. The pulse-to-pulse interaction gives intrachannel cross-phase modulation (IXPM) and four-wave mixing (IFWM) [6], [7]. As a constant pulse train, IXPM induces identical phase modulation and timing jitter to all pulses and does not affect DPSK signal. However, IFWM adds ghost pulses to each DPSK RZ pulse [8]-[11]. The DPSK signal also has higher tolerance to IFWM than OOK signal [11].

When the IFWM-induced ghost pulses are evaluated numerically, the error probability of the DPSK signal can be calculated semianalytically. This letter studies the statistical properties of IFWM in more detail using a method similar to [9] and [11]. Both the error probability and signal-to-noise ratio (SNR) penalty are calculated.

\section{STATISTICS OF IFWM}

If the signal launched to the fiber link is Gaussian pulse train with initial $1 / e$-pulsewidth of $T_{0}$ or full-width at half-maximum pulsewidth of $1.66 T_{0}$, for a bit interval of $T$, the $k$ th pulse is

Manuscript received October 26, 2004; revised December 11, 2004. This work was supported in part by the National Science Council of Taiwan under Grant NSC-93-2213-E-002-061 and Grant NSC92-2218-E-002-055.

The author is with the Institute of Communication Engineering and Department of Electrical Engineering, National Taiwan University, Taipei 106, Taiwan (e-mail: kpho@cc.ee.ntu.edu.tw).

Digital Object Identifier 10.1109/LPT.2005.843942

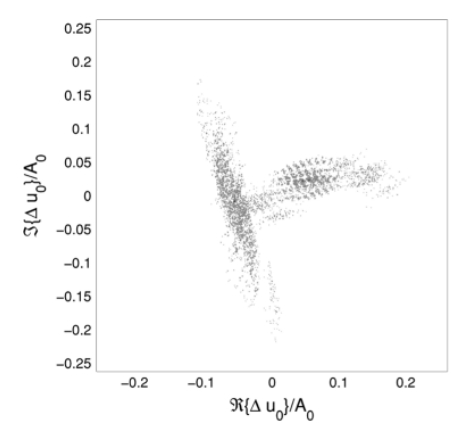

(a)

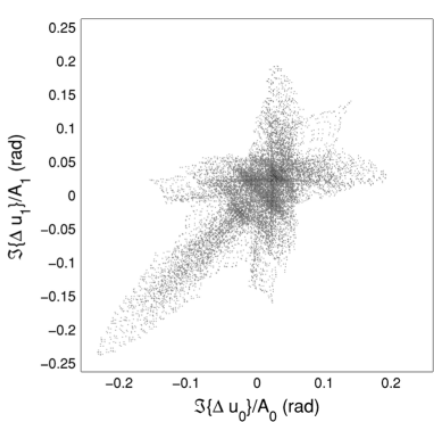

(b)
Fig. 1. (a) Distribution of the complex electric field of $\Delta u_{0} / A_{0}$. (b) Distribution of $\Im\left\{\Delta u_{0}\right\} / A_{0}$ versus $\Im\left\{\Delta u_{1}\right\} / A_{1}$ for two consecutive bit interval.

$u_{k}=A_{k} \exp \left[-(t-k T)^{2} / 2 T_{0}^{2}\right]$, where $A_{k}= \pm A_{0}$ is phase modulated by either 0 or $\pi$. From [8], [9], and [11], the peak amplitude of the ghost pulses induced from IFWM, from the $m, n$, and $(m+n)$ th pulses to the pulse at $t=0$, is

$$
\begin{aligned}
\Delta u_{m, n}= & i \gamma A_{m} A_{n} A_{m+n}^{*} \\
& \times \int_{0}^{L} \frac{e^{-\alpha z}}{\sqrt{1+2 j \beta_{2} z / T_{0}^{2}+3\left(\beta_{2} z / T_{0}^{2}\right)^{2}}} \\
& \times \exp \left\{-\frac{3 m n T^{2}}{T_{0}^{2}+3 j \beta_{2} z}\right. \\
& \left.\quad-\frac{(m-n)^{2} T^{2}}{T_{0}^{2}\left[1+2 j \beta_{2} z / T_{0}^{2}+3\left(\beta_{2} z / T_{0}^{2}\right)^{2}\right]}\right\} d z
\end{aligned}
$$

where $m \neq 0$ and $n \neq 0, \gamma$ is the nonlinear coefficient and $\alpha$ is the attenuation coefficient of the fiber, $\beta_{2}$ is the coefficient of group velocity dispersion, and $L$ is the fiber length per span. Here, we exclude both IXPM with either $m=0$ or $n=0$ and self-phase modulation with $m=n=0$.

Fig. 1(a) shows the distribution of the normalized complex electric field of $\Delta u_{0} / A_{0}$ with the unit of radian. Fig. 1(b) shows the distribution of the peak phase shift of $\Im\left\{\Delta u_{0}\right\} / A_{0}$ versus $\Im\left\{\Delta u_{1}\right\} / A_{1}$ between two consecutive time intervals, where $\Delta u_{0}$ and $\Delta u_{1}$ are the peak-amplitude of ghost pulses and $\Im\{\cdot\}$ is the imaginary part of a complex number. The ghost pulses of $\Delta u_{0}$ and $\Delta u_{1}$ include all contributions of $-8<m, n, m+n \leq$ 8 for a 16-bit DPSK signal with about 64000 combinations. Fig. 1(a) is for ghost pulse at the center bit and Fig. 1(b) is 
for the center two bits. If the pulse amplitude of $\left|A_{0}\right|$ is significantly larger than the IFWM ghost pulses, $\Im\left\{\Delta u_{0}\right\} / A_{0}$ and $\Im\left\{\Delta u_{1}\right\} / A_{1}$ give approximately the phase shift [11].

Fig. 1(a) and (b) is obtained for an $N$-span fiber link with $L=100 \mathrm{~km}$ of fiber per span with a normalized launched power of unity mean nonlinear phase shift of $\left\langle\Phi_{\mathrm{NL}}\right\rangle=N \gamma L_{\text {eff }} P_{0}=$ $1 \mathrm{rad}$, where $P_{0}=\sqrt{\pi}\left|A_{0}\right|^{2} T_{0} / T$ is the launched power, and $L_{\text {eff }} \approx 1 / \alpha$ is the effective nonlinear length per span. $N$ identical fiber spans are repeated one after another with $100 \%$ dispersion compensation at the end of each fiber span. For arbitrary fiber link configuration, $N$ instead of one integration of (1) are required. IFWM ghost pulses add coherently span after span as the worst case. The fiber link has an attenuation coefficient of $\alpha=0.2 \mathrm{~dB} / \mathrm{km}$. With a bit interval of $T=25 \mathrm{ps,}$ DPSK signal has a data rate of $40 \mathrm{~Gb} / \mathrm{s}$. The initial pulsewidth is $T_{0}=5 \mathrm{ps}$, for a duty cycle of about $1 / 3$. The fiber dispersion is $\beta_{2}=-22 \mathrm{ps}^{2} / \mathrm{km}$, corresponding to $D=17 \mathrm{ps} / \mathrm{km} / \mathrm{nm}$ at the wavelength of $1.55 \mu \mathrm{m}$ for standard single-mode fiber.

The distribution of Fig. 1(a) is very irregular and has significant discrepancy with Gaussian distribution. Similar to Wei and Liu [11, Fig. 2], Fig. 1(b) is only symmetrical with respect to $x=y$. The difference of Fig. 1(b) with [11] is for a lossy instead of lossless fiber. With span by span dispersion compensation and for lossy fiber, 50\% precompensation of dispersion increases instead of reduces IFWM. The phase of $\Im\left\{\Delta u_{0}\right\} / A_{0}$ is correlated with $\Im\left\{\Delta u_{1}\right\} / A_{1}$ with a correlation coefficient of about 0.58 . For $N$ repeated identical fiber spans, Fig. 1(a) and (b) is valid for single-span and multispan systems with $\left\langle\Phi_{\mathrm{NL}}\right\rangle=1 \mathrm{rad}$. Note that both $\Delta u_{0} / A_{0}$ and $\Delta u_{1} / A_{1}$ are zero mean. Not shown in Fig. 1, the real parts of $\Re\left\{\Delta u_{0}\right\} / A_{0}$ and $\Re\left\{\Delta u_{1}\right\} / A_{1}$ have a correlation coefficient of about -0.54 .

\section{ERROR PROBABILITY FOR DPSK SIGNALS}

The error probability of DPSK signals with IFWM is difficult to find analytically. From Fig. 1, the distribution of the IFWM-induced ghost pulses is not Gaussian distributed. With the distribution of Fig. 1, the error probability of DPSK signal with IFWM can be calculated semianalytically.

Assumed for simplicity that the transmitted phases at $t=0$ and $t=T$ are identical and, without loss of generality, the transmitted signals are $E_{s}(t)=E_{s}(t-T)=A_{0}>0$. With optical amplifier noise of $n(t)$, ignoring the constant factor of interferometer loss and photodiode responsivity, the photocurrent is [12]

$$
\begin{aligned}
i(t)=\mid 2 A_{0}+\Delta u_{1} & +\Delta u_{0}+n(t)+\left.n(t-T)\right|^{2} \\
& -\left|\Delta u_{1}-\Delta u_{0}+n(t)-n(t-T)\right|^{2} .
\end{aligned}
$$

A decision error occurs if $i(t)<0$.

Given $\Delta u_{0}$ and $\Delta u_{1}$, the two terms in (2) are independent of each other and have a noncentral chi-square distribution [13, pp. 41-44]. Each term of (2) has the same noise variance of $4 \sigma_{n}^{2}$, where $E\left\{|n(t)|^{2}\right\}=2 \sigma_{n}^{2}$ with $\sigma_{n}^{2}$ as the noise variance per dimension. The noncentralities of the two terms of (2) are $\left|2 A_{0}+\Delta u_{1}+\Delta u_{0}\right|^{2}$ and $\left|\Delta u_{1}-\Delta u_{0}\right|^{2}$, respectively. From [13, Appendix B] and [14], the probability of $i(t)<0$ is equal to

$$
p_{e}\left(\Delta u_{0}, \Delta u_{1}\right)=Q(a, b)-\frac{1}{2} e^{-\left(a^{2}+b^{2}\right) / 2} I_{0}(a b)
$$

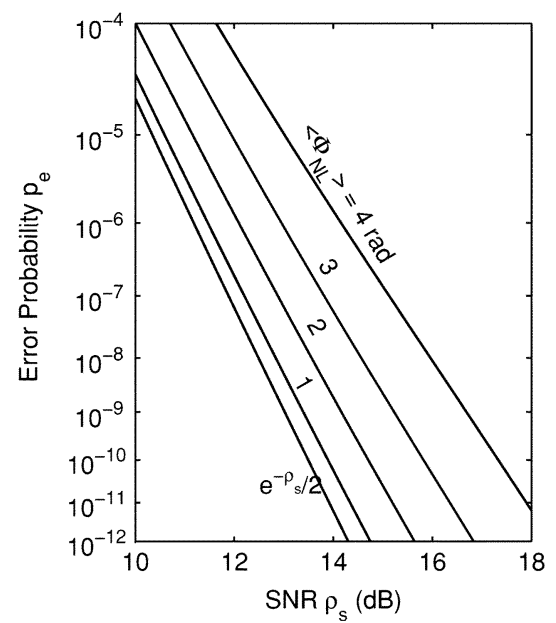

Fig. 2. Error probability of DPSK signals with IFWM ghost pulses as a function of SNR $\rho_{s}$.

where $Q(\cdot, \cdot)$ is the Marcum $Q$-function and

$$
\begin{aligned}
& a^{2}=\frac{\rho_{s}}{2}\left|2+\frac{\Delta u_{1}}{A_{0}}+\frac{\Delta u_{0}}{A_{0}}\right|^{2} \\
& b^{2}=\frac{\rho_{s}}{2}\left|\frac{\Delta u_{1}}{A_{0}}-\frac{\Delta u_{0}}{A_{0}}\right|^{2}
\end{aligned}
$$

where $\rho_{s}=A_{0}^{2} / 2 \sigma_{n}^{2}$ is the SNR without taking into account the ghost pulses. Evaluated semianalytically using the distribution of Fig. 1(a) and (b), the error probability is equal to

$$
p_{e}=E\left\{p_{e}\left(\Delta u_{0}, \Delta u_{1}\right)\right\}
$$

where $E\{\cdot\}$ denotes expectation.

When the sequence of $A_{k}$ is changed to $(-1)^{k} A_{k}$ with all odd positions changing sign, from (1), $\Delta u_{0}$ remains the same but $\Delta u_{1}$ changes sign. As $\Delta u_{0} / A_{0}$ and $\Delta u_{1} / A_{1}$ remain the same for Fig. 1(b), the error probability for the case with $A_{0}=-A_{1}$ is the same as that of (6).

Fig. 2 shows the error probability as a function of SNR $\rho_{s}$ for DPSK signal with IFWM-induced ghost pulses. The error probability without IFWM of $(1 / 2) e^{-\rho_{s}}[13$, Sec. 5.2.8] is also shown for comparison. The semianalytical formula of (6) with (3) is used to calculate the error probability based on IFWM ghost pulse distribution of Fig. 1.

Fig. 3 shows the SNR penalty for $p_{e}=10^{-9}$ as a function of the mean nonlinear phase shift of $\left\langle\Phi_{\mathrm{NL}}\right\rangle$. In addition to the SNR penalty corresponding to Fig. 2, Fig. 3 also shows the penalty when the initial pulsewidth is $T_{0}=7.53 \mathrm{ps}$ for a duty cycle of $1 / 2$. For $1-\mathrm{dB}$ SNR penalty, the mean nonlinear phase shift must be less than 1.25 and $1.80 \mathrm{rad}$ for initial pulsewidth of $T_{0}=5$ and $7.53 \mathrm{ps}$, respectively. The SNR penalty is smaller for small initial pulsewidth of $T_{0}=5 \mathrm{ps}$.

If the IFWM-induced ghost pulses are assumed as Gaussian distributed, the noise increases to $n(t)+\Delta u_{0}$ at $t=0$. However, the SNR of $\left|A_{0}\right|^{2} / E\left\{\left|n(t)+\Delta u_{0}\right|^{2}\right\}$ cannot be used directly to find the error probability due the correlation between the ghost pulses of $\Delta u_{0}$ and $\Delta u_{1}$. The phase variance of $A_{0}+n(t)$ is equal to about $1 / 2 \rho_{s}$ [13, Sec. 5.2.7]. The variance of the differential phase is $1 / \rho_{s}$ and should compare with

$$
\sigma_{\delta \theta}^{2}=E\left\{\left[\Im\left\{\Delta u_{0} / A_{0}\right\}-\Im\left\{\Delta u_{1} / A_{1}\right\}\right]^{2}\right\}
$$




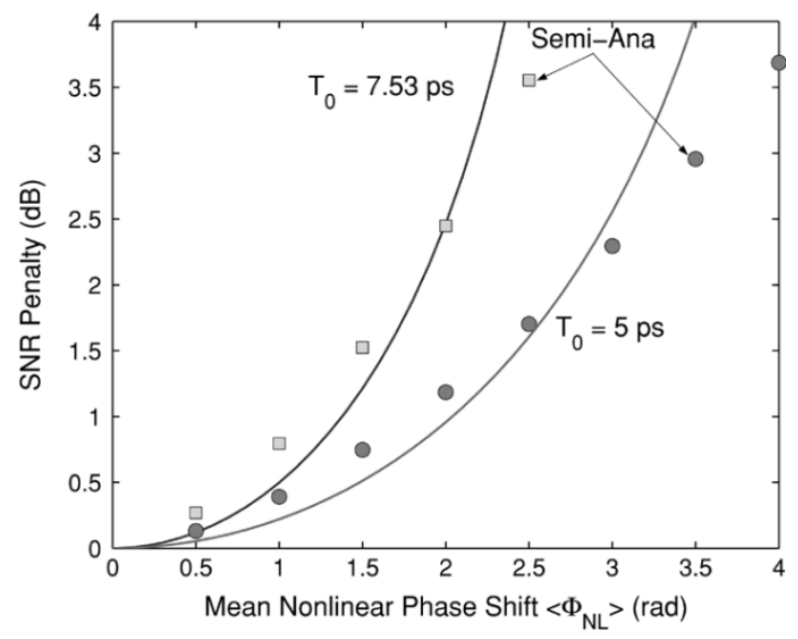

Fig. 3. SNR penalty versus mean nonlinear phase shift of $\left\langle\Phi_{\mathrm{NL}}\right\rangle$. The curves are from empirical formula.

due to IFWM ghost pulses. The SNR penalty can be empirically estimated as $-10 \cdot \log _{10}\left(1-20 \sigma_{\delta \theta}^{2}\right)$, where 20 is the SNR for a DPSK error probability of $10^{-9}$. This empirical formula finds the SNR penalty based on the variance of the differential phase. Fig. 3 also shows the SNR penalty from the empirical approximation. For SNR penalty less than $2 \mathrm{~dB}$, the empirical approximation underestimates the SNR penalty by less than $0.25 \mathrm{~dB}$. For SNR penalty larger than $2 \mathrm{~dB}$, the approximation overestimates the SNR penalty.

The empirical approximation still requires extensive numerical calculations to find the variance of $\sigma_{\delta \theta}^{2}$ from many combinations of bit sequence. The semianalytical method needs one further step to find the error probability of (3) for each term of $\Delta u_{0}$ and $\Delta u_{1}$, and then averaging of (6).

The above analysis and numerical results always used the peak amplitude of the ghost pulses and the signal pulses. The pulsewidth of the ghost pulses is ignored for simplicity. However, the IFWM ghost pulse broadens to $\sqrt{3}$ times the signal pulsewidth [8], [9]. As the power is proportional to the pulsewidth, the ghosts pulse has $\sqrt{3}$ times larger energy than the signal for the same peak amplitude. In the worst case, the $x$ axis of Fig. 3 must scale by a factor of $3^{1 / 4}=1.32$. However, the scale factor depends on the bandwidth of the optical and electrical filters in the receiver.

If the optical matched filter precedes the direct-detection DPSK receiver and the electric filter at the receiver has a wide bandwidth that does not distort the signal, the IFWM amplitude is increased by a factor of $\sqrt{3 / 2}=1.22$ by the receiver, slightly less than the ratio of 1.32 . If both the optical and electrical filters have a very wide bandwidth, allowing too much noise to the receiver, the peak amplitude directly transfers to the receiver. If the optical filter has a wide bandwidth but the electrical filter is a $0.75 / T$ bandwidth Bessel filter, IFWM increases by a factor of 1.19 and 1.33 for $T_{0}=7.53$ and $5 \mathrm{ps}$, respectively. In practical system design, Fig. 3 must be modified to take into account the design of both receiver and transmitter. Note that the mean nonlinear phase shift of Fig. 3 is a simple system parameter to evaluate.
Fig. 3 shows that DPSK signal with IFWM can tolerate a far larger mean nonlinear phase shift of $\left\langle\Phi_{\mathrm{NL}}\right\rangle$ than DPSK signal with nonlinear phase noise of $\left\langle\Phi_{\mathrm{NL}}\right\rangle<0.6 \mathrm{rad}$ for 1 -dB penalty [15]. However, Ho [15] is for nonreturn-to-zero signal without pulse distortion and deduces that RZ signal has lower tolerance to nonlinear phase noise. We are currently developing a model for RZ signal with nonlinear phase noise in highly dispersive systems for a fair comparison.

\section{CONCLUSION}

When the peak amplitude of IFWM-induced ghost pulses is evaluated numerically, the error probability of DPSK signals can be found semianalytically. For an SNR penalty less than $1 \mathrm{~dB}$, the mean nonlinear phase shift of the system must be less than 1 to 2 rad depending on the initial pulsewidth. An empirical approximation is also used to find the SNR penalty up to $2 \mathrm{~dB}$.

\section{REFERENCES}

[1] A. H. Gnauck et al., " $2.5 \mathrm{~Tb} / \mathrm{s}(64 \times 42.7 \mathrm{~Gb} / \mathrm{s})$ transmission over $40 \times 100 \mathrm{~km}$ NZDSF using RZ-DPSK format and all-Raman-amplified spans," presented at the Opt. Fiber Commun. Conf., Anaheim, CA, 2002, Postdeadline Paper FC2.

[2] B. Zhu et al., "6.4- Tb/s $(160 \times 42.7 \mathrm{~Gb} / \mathrm{s})$ transmission with $0.8 \mathrm{bit} / \mathrm{s} / \mathrm{Hz}$ spectral efficiency over $32 \times 100 \mathrm{~km}$ of fiber using CSRZ-DPSK format," presented at the Opt. Fiber Commun. Conf., Atlanta, GA, 2003, Postdeadline Paper PD19.

[3] C. Rasmussen et al., "DWDM 40G transmission over trans-Pacific distance $(10000 \mathrm{~km})$ using CSRZ-DPSK, enhanced FEC and all-Raman amplified $100 \mathrm{~km}$ ultra-wave fiber spans," presented at the Opt. Fiber Commun. Conf., Atlanta, GA, 2003, Postdeadline Paper PD18.

[4] J.-X. Cai et al., "RZ-DPSK field trial over $13100 \mathrm{~km}$ of installed non slope-matched submarine fibers," presented at the Opt. Fiber Commun. Conf., Anaheim, CA, 2004, Postdeadline Paper PDP34.

[5] C. Xu, X. Liu, and X. Wei, "Differential phase-shift keying for high spectral efficiency optical transmissions," IEEE J. Sel. Topics Quantum Electron., vol. 10, no. 2, pp. 281-293, Mar./Apr. 2004.

[6] I. Shake, H. Takara, K. Mori, S. Kawanishi, and Y. Yamabayashi, "Influence of inter-bit four-wave mixing in optical TDM transmission," Electron. Lett., vol. 34, no. 16, pp. 1600-1601, 1998.

[7] R.-J. Essiambre, B. Mikkelsen, and G. Raybon, "Intra-channel crossphase modulation and four-wave mixing in high-speed TDM systems," Electron. Lett., vol. 35, no. 19, pp. 1576-1578, 1999.

[8] A. Mecozzi, C. B. Clausen, and M. Shtaif, "Analysis of intrachannel nonlinear effects in highly dispersed optical pulse transmission," IEEE Photon. Technol. Lett., vol. 12, no. 4, pp. 292-294, Apr. 2000.

[9] A. Mecozzi, C. B. Clausen, M. Shtaif, S.-G. Park, and A. H. Gnauck, "Cancellation of timing and amplitude jitter in symmetric links using highly dispersed pulses," IEEE Photon. Technol. Lett., vol. 13, no. 5, pp. 445-447, May 2001

[10] M. J. Ablowitz and T. Hirooka, "Resonant intrachannel pulse intraction in dispersion-managed transmission systems," IEEE J. Sel. Topics Quantum Electron., vol. 8, no. 3, pp. 603-614, May/Jun. 2002.

[11] X. Wei and X. Liu, "Analysis of intrachannel four-wave mixing in differential phase-shift keying transmission with large dispersion," Opt. Lett., vol. 28, no. 23, pp. 2300-2302, 2003.

[12] K.-P. Ho, "The effect of interferometer phase error on direct-detection DPSK and DQPSK signals," IEEE Photon. Technol. Lett., vol. 16, no. 1, pp. 308-310, Jan. 2004.

[13] J. G. Proakis, Digital Communications, 4th ed. New York: McGrawHill, 2000.

[14] S. Stein, "Unified analysis of certain coherent and noncoherent binary communications systems," IEEE Trans. Inf. Theory, vol. IT-10, no. 1, pp. 43-51, Jan. 1964.

[15] K.-P. Ho, "Error probability of DPSK signals with cross-phase modulation induced nonlinear phase noise," IEEE J. Sel. Topics Quantum Electron., vol. 10, no. 2, pp. 421-427, Mar./Apr. 2004. 